free two-photon experiments (the Laboratoire de Spectroscopie Hertzienne in Paris reported on a doppler-free three-quantum experiment), in saturation spectroscopy experiments, in beam foil experiments...

Despite the large number of applications of lasers in atomic spectroscopy one has the impression that not all the possibilities offered by laser spectroscopy have yet been explored by the atomic physicist. Perhaps, at the next EGAS conference which will be held in Cracow, Poland, 12-15 July, 1977, we shall learn more about this topic. Preliminary registration for this conference should incidentally be made by Oct. 31 because of difficulties in reserving accommodation.

H. Hühnermann

\section{Atomic Physics Division}

The Board Members of the Atomic Spectroscopy Section (EGAS) from July 1976-77 are as follows :

D.N. Stacey, Clarendon Lab., Oxford (chairman)

E. Meinders, Zeeman Lab., Amsterdam Univ. (secretary)

T. Anderson, Inst. of Phys., Aarhus

J. Bauche, Aimé Cotton Lab., Orsay

W.R.S. Garton, Imperial Coll., London

H. Hühnermann, University, Marburg

M.D. Kunisz, Jagellonian Univ., Cracow

J.C. Lehmann, Herz Spectroscopy Lab., Paris VI Univ.

I. Martinson, Inst. of Physics, Lund University

R. Winkler, Optics Inst., Berlin T.U.

\section{Condensed Matter Division}

The Third General CMD Meeting, following those in Florence (1971) and Budapest (1974) will be held at Leeds, UK, 26-29 July, 1977. As in the previous meetings two topics will be discussed, one related to a phenomenon of interest to all sections of the division: "Electron transport in condensed matter"

and one related to a class of materials: "Molecular solids including polymers, liquid crystals, 1- and 2-dimensional solids".
The deadlines for contributed papers are :

1 October, 1976

(preliminary intentions)

1 February, 1977

(final abstract)

Further information can be obtained from the Meetings Officer, The Institute of Physics, 47 Belgrave Square, London SW1X 8QX. Abstracts of contributions should be sent to Professor G.J. Morgan, Department of Physics, University of Leeds, Leeds LS2 9JT.

\section{Joint European Torus}

\section{Appointment of the Head of Project}

The post of Head of Project (director) of the Joint European Torus (JET) is to be created on 1st January 1977.

JET is the largest plasma confinement experiment in the current five-year European Community fusion research programme. It is a tokamak designed to produce plasmas of the type needed for the thermo-nuclear reactors and for experiments on them. Its construction is planned to take five years.

The design of this machine is largely completed, and a decision is expected shortly on its construction, and on its location within the European Communities. JET has been designed by a team bringing together the experience of all fusion research laboratories associated with the European Community, and these organizations will co-operate in its construction and operation.

The Head of Project will take executive responsibility for the successful construction and operation of JET. He will be responsible to a Community management committee and will lead a multinational team.

$\mathrm{He}$ must have leadership and management experience, and a wide professional competence preferably including both engineering and experimental physics. $\mathrm{He}$ will receive support in fusion-physics and technology, engineering and administration (including financial and personnel expertise) but he should himself have a wide enough experience to form his own judgments.

The salary will be in the $A / 2$ range of staff of the Commission of the European Communities. The appointment will last for five years with the prospect of renewal. The candidate will be expected to work outside his country.

Applications, which should be accompanied by a curriculum vitae, with names of two referees, should be sent by November 15th., 1976 to:

$$
\begin{gathered}
\text { The Secretary, JET Management Committee } \\
\text { Fusion Directorate, DG XII } \\
\text { Commission of the European Communities } \\
\text { rue de la Loi, } 200 \text { B-1049 Brussels }
\end{gathered}
$$

Further information can be obtained from the same address.
We apologize for the inversion of the chairman and secretary in the directory section of the June issue of Europhysics News.

The composition of the Board of the Molecular Physics Section has now been confirmed. It is identical to that of the provisional board listed in the directory section of the June issue.

\section{Reduced Rates for Individual Ordinary Members}

Individual Ordinary Members of EPS benefit from reduced rates of inscription to many conferences. Details of these rates are given in the list of Meetings published in the yellow issues of $E N$.

The Institute of Physics announces the special rates to EPS Individual Ordinary Members in 1977 for their personal copies of the following publications (normal rates are shown in brackets)

Physics Bulletin : $£ 7.50$ ( $£$ 18.00)

Physics Education : $₹ 6.25$ ( $₹ 14.00)$

Physics in Technology: $£ 5.00$ ( $£ 20.00$ )

The American Institute of Physics offers to Individual Ordinary Members and to members of Member Societies for their personal use the following special rates:

Physics Today: surface mail, \$16 (\$30); airmail, \$20 (\$ 34)

Current Physics Index: surface, \$ 55 (\$102); airmail, \$ 62 (\$110)

The publishers of the interdisciplinary journal of the Ettore Majorana Centre, Progress in Scientific Culture, announce the special rate of $95 \mathrm{Sw}$. Fr./an. for EPS Individual Ordinary Members for personal use, compared with $120 \mathrm{Sw}$. Fr./an. for other individuals and $160 \mathrm{Sw}$. Fr./an.. for institutions. Orders through EPS secretariat.

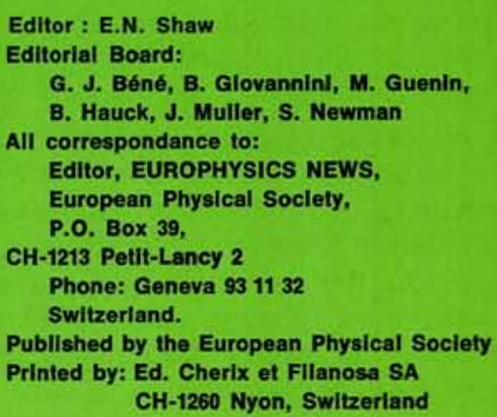

